\title{
A cross-sectional survey of factors influencing bone mass in junior high school students
}

\author{
Yuka Tamura • Isao Saito • Yasuhiko Asada • \\ Taro Kishida • Masamitsu Yamaizumi • \\ Tadahiro Kato
}

Received: 18 June 2012/Accepted: 14 November 2012/Published online: 8 December 2012

(C) The Japanese Society for Hygiene 2012

\begin{abstract}
Objective The purpose of this study is to investigate factors influencing the osteo-sono assessment index (OSI) in junior high school students (boys, girls who had reached menarche, and girls who had not).

Methods A total of 9,743 students (4,974 boys and 4,769 girls) in Ehime Prefecture participated in this study. We measured body mass index (BMI) and calcaneal bone mass using OSI. In parallel, participants answered a questionnaire relating to age, sex, menarche, exercise habits, milk intake, and history of bone fractures during the preceding year. To determine the factors influencing OSI, we calculated an individual standardized partial regression coefficient $(\beta)$ using multiple linear regression (MLR) analysis.
\end{abstract}

Y. Tamura · Y. Asada · T. Kishida · T. Kato

Department of Applied Bioresource Science,

The United Graduate School of Agricultural Sciences,

Ehime University, 3-5-7 Tarumi, Matsuyama,

Ehime 790-8566, Japan

Y. Tamura $(\bowtie)$

Department of Applied Bioresource Sciences,

The United Graduate School of Agricultural Sciences,

Ehime University, 1-5-22 Mochida-cho, Matsuyama,

Ehime 790-0855, Japan

e-mail: tamura.yuka.my@ehime-u.ac.jp

I. Saito

Department of Basic Nursing and Health Science,

Graduate School of Medicine, Ehime University,

454 Shitsukawa, Toon, Ehime 791-0295, Japan

M. Yamaizumi - T. Kato

Ehime Prefectural Federation of Agricultural Cooperatives for Health and Welfare (JA Ehime Kouseiren),

533-1 Takanoko-cho, Matsuyama, Ehime 790-0925, Japan
Results For boys, MLR showed that BMI $(\beta=0.300)$, age $(\beta=0.260)$, current exercise habits $(\beta=0.106)$, and milk intake per day in primary school $(\beta=0.085)$ statistically significantly influenced OSI. For girls who had reached menarche, BMI $(\beta=0.302)$, current exercise habits $(\beta=0.237)$, age $(\beta=0.140)$, and bone fracture during the preceding year $(\beta=0.036)$ influenced OSI. For girls who had not reached menarche, current exercise habits $(\beta=0.242)$, BMI $(\beta=0.135)$, and age $(\beta=0.085)$ influenced OSI.

Conclusions There were differences between the factors related to OSI among boys, girls who had reached menarche, and girls who had not. BMI, exercise habits, and age were the common factors related to OSI. Particularly for girls, exercise habits had a great influence on OSI.

Keywords OSI $\cdot$ Junior high school students $\cdot$ Menarche Bone fracture $\cdot$ Exercise habits

\section{Introduction}

Recent studies have reported that height, weight, BMI, exercise habits, and milk intake are factors relating to the development of bone mass in junior high school students [1-3]. The incidence of bone fractures in Japanese primary, junior, and senior high schools has been increasing in recent years $[4,5]$. Bone fractures during puberty are not only a disability affecting school and daily life; they also potentially inhibit normal bone growth [6].

Secondary sexual development begins during junior high school, resulting in the secretion of an increased level of sex hormones. Kokudo et al. [7] reported that it is during junior high school that differences in bone characteristics between boys and girls, including bone mass and bone 
strength, become apparent. Some reports have suggested that bone mass in girls who have reached menarche is higher than that in girls who have not reached menarche [2, 8 ,9]. Because bone mass during junior high school is influenced by secondary sexual development, it is conceivable that the effect on bone mass will differ between boys and girls, and also be affected by whether or not girls have reached menarche. However, few studies have compared factors that influence the development of bone mass, such as physique, lifestyle, and history of bone fractures, among boys, girls who have reached menarche, and girls who have not reached menarche.

The objective of this study is to investigate factors influencing the development of bone mass in junior high school students (boys, girls who had reached menarche, and girls who had not reached menarche). In addition, as a resource for school health education professionals, we present basic statistical data regarding the causes of bone fractures.

\section{Methods}

\section{Participants}

As of 2009, Ehime Prefecture had 144 junior high schools (140 public schools including one national school, and 4 private schools) with a total of 39,902 students. Ehime Prefecture is divided into three broad geographic areas. The Ehime Prefecture Board of Education selected 57 public junior high schools for this study, selecting 19 schools from each of the three geographical regions. In 2009, $99.3 \%$ of public junior high schools and $99.7 \%$ of public primary schools provided school lunch in Ehime Prefecture [10]. School lunch was provided at all 57 of the public junior high schools surveyed in this report. School lunch consisted of bread or rice plus side dishes and always included a $200 \mathrm{~mL}$ serving of milk.

Between 7 November 2007 and 17 February 2009 we surveyed 10,418 students from these 57 junior high schools. A total of 9,987 students (excluding 431 students who were absent on the day of the medical examination) underwent bone mass measurement and answered our questionnaire. To ensure consistency and maximize the number of questionnaires returned, school nurses and medical examination staff reviewed the questionnaire forms to check for incomplete answers and helped students to fill in the questionnaire fully where necessary. Despite these efforts, 223 respondents did not fill out the questionnaire correctly or filled it out incompletely. We obtained bone mass data and completed questionnaires from 9,764 students. The final response rate for our survey was $93.7 \%$. Medical records kept at school indicated that
21 students had been suffering from disorders that interfere with normal growth, such as short stature, severe scoliosis (including adolescent idiopathic scoliosis), bone dysplasia, and anorexia nervosa requiring psychiatric treatment. Accordingly we excluded these 21 students and analyzed the data derived from the remaining 9,743 (93.5\%) students (4,974 boys and 4,769 girls). The 9,743 students in our survey represent approximately $24.4 \%$ of the total number of junior high school students in Ehime Prefecture.

The Ehime Prefecture Board of Education explained our study protocol to individual junior high school principals. Homeroom teachers and school nurses informed students and their parents about the study. Participants and their parents provided written informed consent before starting the study. The questionnaire was located on the reverse side of the survey consent form signed by individual students and their parents. The Ethics Committee of Ehime Prefectural Federation of Agricultural Cooperatives for Health and Welfare (JA Ehime Kouseiren) approved this study prior to its implementation.

\section{Medical examination and questionnaire}

In Japan, height and weight are measured during annual medical examinations carried out in junior high schools. Height and weight were measured using a digital weight scale (Tanita Co.). Body mass index (BMI) was calculated using body weight in kilograms divided by the square of height in meters.

Measurement of bone mass during annual medical examinations is rare. Bone mass was indicated by the osteo-sono assessment index (OSI) which we calculated using quantitative ultrasound (QUS). We suggest that using QUS to measure OSI has several benefits: (1) it does not use ionizing radiation; (2) it is easy to use; (3) it is relatively inexpensive; and (4) it is highly correlated (0.5-0.8 correlation coefficient) with dual X-ray absorptiometry measured bone mass values [11]. Calcaneal bone OSI was calculated based on the speed of sound (SOS) and transmission index (TI) - half the maximum amplitude of the sound wave as measured by the Acoustic Osteo-Screener ultrasound device (AOS-100; ALOKA Co., Japan). OSI was calculated according to the following formula: $\mathrm{OSI}=\mathrm{TI} \times(\mathrm{SOS})^{2}$. The OSI values used in the text, tables, and figures in this report were all divided by $10^{6}$.

We also investigated the following factors: age, sex, exercise habits during primary school and currently ("currently" means "at the time of answering the questionnaire"), milk intake during primary school and currently, whether or not the participant had suffered a bone fracture during the preceding year, causes of bone fractures, where on the body bone fractures had occurred, and, in the case of girls, whether or not they had undergone 
menarche. Milk intake was broken down into two categories: (1) milk intake per day when school lunch was provided, and (2) milk intake per day when school lunch was not provided. We calculated average milk intake per day during primary school and currently, using the following formula: average milk intake $=$ (milk intake per day $\times 5$ days when school lunch was provided + milk intake per day $\times 2$ days when school lunch was not provided) $/ 7$.

\section{Statistical analysis}

Unpaired $t$ test and one-way analysis of variance (ANOVA) were performed to determine the significance in the difference between means. Chi-square test was performed to determine whether or not two categorical variables have association. BMI values were classified as follows: underweight $\left(18.4 \mathrm{~kg} / \mathrm{m}^{2}\right.$ or lower), normal range $\left(18.5-24.9 \mathrm{~kg} / \mathrm{m}^{2}\right)$, and obese $\left(25.0 \mathrm{~kg} / \mathrm{m}^{2}\right.$ or greater) based on the standard of the Japanese Society for the Study of Obesity [12]. Exercise habits were divided into three categories: (1) exercised during primary school and currently, (2) exercised either during primary school or currently, (3) did not exercise either during primary school or currently. Milk intake per day was classified into three categories (1) $<200 \mathrm{~mL}$, (2) between 200 and $400 \mathrm{~mL}$, (3) $>400 \mathrm{~mL}$ by reference to an adequate daily milk intake (200-400 $\mathrm{mL}$ per day) recommended by The Japan Dietetic Association [13]. Experiencing bone fracture during the preceding year was divided into two categories.

To determine the factors that influenced OSI, we performed multiple linear regression (MLR) analyses using the forced entry method for the independent factor (OSI) and dependent factors such as age, BMI, exercise habits, milk intake, and whether or not bone fracture occurred during the preceding year. Both exercise habits and bone fractures were used for the dummy variables $(1$ or 0$)$ in the forced entry method of MLR analysis, as follows: ' 1 ' is 'engages in exercise,' ' 0 ' is 'does not engage in exercise,' ' 1 ' is 'has experienced a bone fracture during the preceding year,' ' 0 ' is 'has not experienced a bone fracture during the preceding year.' Statistically significant difference was defined as $5 \%$ or less for all analyses using SPSS version 19 (IBM Japan).

\section{Results}

The participants in this study comprised junior high school students, of which 4,974 were boys, 4,184 were girls who had reached menarche, and 585 were girls who had not reached menarche.
Table 1 presents age brackets, height, weight, BMI, OSI, milk intake, exercise habits, and number of students who had suffered bone fracture during the preceding year. As supplemental data, during primary school, 206 students [62 boys and 144 girls $(2.1 \%)$ ] did not drink milk $(0 \mathrm{~mL}$ milk intake) on days when school lunch was provided and 3,520 students [1,550 boys and 1,970 girls (36.1\%)] did not drink milk ( $0 \mathrm{~mL}$ milk intake) on days when school lunch was not provided. During junior high school, 105 students [48 boys and 57 girls (1.1\%)] did not drink milk ( $0 \mathrm{~mL}$ milk intake) on days when school lunch was provided and 3,479 students [1,542 boys and 1,937 girls (35.7\%)] did not drink milk ( $0 \mathrm{~mL}$ milk intake) on days when school lunch was not provided. In our study, 380 boys (7.6\%), 980 girls who had reached menarche (23.4\%), and 103 girls who had not reached menarche $(17.6 \%)$ had not exercised regularly since primary school (Table 1). The percentage of girls who did not exercise was higher than that of boys. There were 437 boys and 216 girls who had suffered a bone fracture during the preceding year. There were a total of 479 bone fractures among boys and 230 among girls in the preceding year (Table 2). The most common bone fractures, for both boys and girls, were fractures of the finger, wrist, and foot. These three fractures were frequently caused by sports such as soccer, baseball, and basketball (Table 3). As $69.5 \%$ of total fracture locations were caused by sports, we examined the relationship between exercise habits and bone fractures. For boys, there was no significant correlation between current exercise habits and experiencing a bone fracture in the preceding year, using chi-square test $\left(\chi^{2}\right.$ value $=2.71$, $P=0.990)$. On the other hand, for girls, there was a significant correlation between current exercise habits and experiencing a bone fracture in the preceding year, using chi-square test (girls who had reached menarche: $\chi^{2}$ value $=18.9, P<0.001$; girls who had not reached menarche: $\chi^{2}$ value $=6.4, P=0.011$ ).

Figure 1 shows the average value of OSI according to age and sex (and in addition, for girls, whether or not they had reached menarche). Using ANOVA, we compared the OSI values for three groups: (1) boys, (2) girls who had reached menarche, and (3) girls who had not reached menarche. The average OSI value was highest in girls who had reached menarche, followed by boys and then girls who had not reached menarche. There was no significant difference in OSI value (1) between 12-year-old boys and 12-year-old girls who had not reached menarche, and (2) between 15 -year-old boys and 15-year-old girls who had reached menarche.

Table 4 presents the relationship between average OSI values for boys and the following five factors: (1) BMI, (2) exercise habits, (3) milk intake per day during primary school, (4) current milk intake per day, and (5) whether or 
Table 1 Participant characteristics

Boys $(N=4,974) \quad$ Girls who have reached

menarche $(N=4,184)$
Girls who have not reached menarche $(N=585)$

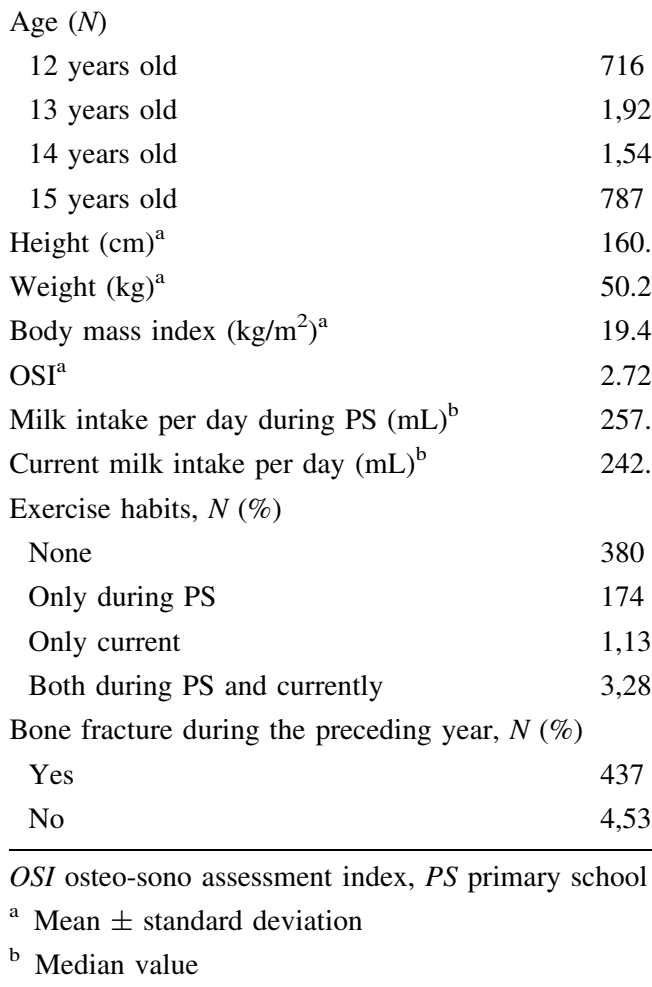

not the participant experienced a bone fracture during the preceding year. In boys, for each age group (12, 13, 14, and 15 years), the average OSI values were significantly different for (1) BMI, (2) exercise habits, and (3) milk intake per day during primary school. For each age group (13, 14, and 15 years but excluding 12 years old), the average OSI values were significantly different for current milk intake per day. Whether or not participants had experienced a bone fracture in the preceding year was not significantly different in OSI in any of the age groups.

Table 5 presents the same relationship for girls who had reached menarche and for girls who had not reached menarche. In girls who had reached menarche, for each age group $(12,13,14$, and 15 years), the average OSI values were significantly different for (1) BMI, (2) exercise habits, and (3) current milk intake per day. For each age group (13, 14 , and 15 years but excluding 12 years old), the average OSI values were significantly different for (1) milk intake per day during primary school and (2) experiencing a bone fracture during the preceding year. In girls who had not reached menarche, the average OSI values were significantly different for (1) BMI, (2) exercise habits, and (3) milk intake per day during primary school. The average OSI values were not significantly different for (1) current milk intake per day and (2) experiencing a bone fracture during the preceding year.

Table 6 presents the standardized partial regression coefficient $(\beta)$ for boys, girls who had reached menarche, and girls who had not reached menarche. Because "current exercise habits" was significantly associated with "exercise habits during primary school" for three groups (boys: $\chi^{2}$ value $=437.17, P<0.001$; girls who had reached menarche: $\chi^{2}$ value $=375.91, P<0.001$; and girls who had not reached menarche: $\chi^{2}$ value $\left.=37.44, P<0.001\right)$, "current exercise habits" (excluding "exercise habits during primary school") was treated as a dependent factor and subjected to multiple regression analysis. For boys, MLR showed that the following factors had a significant influence on OSI, in descending order: $\operatorname{BMI}(\beta=0.300)$, age $(\beta=0.260)$, current exercise habits $(\beta=0.106)$, and milk intake per day in primary school $(\beta=0.085)$. For girls who had reached menarche, MLR showed that the following factors had a significant influence on OSI, in descending order: $\mathrm{BMI}(\beta=0.302)$, current exercise habits $(\beta=0.237)$, age $(\beta=0.140)$, and bone fracture during the preceding year $(\beta=0.036)$. For girls who had not reached menarche, MLR showed that the following factors had a significant influence on OSI, in descending 
Table 2 Total number of incidents of bone fracture during the preceding year

\begin{tabular}{|c|c|c|}
\hline Location of bone fracture & Boys $(N=479)$ & Girls $(N=230)$ \\
\hline \multicolumn{3}{|l|}{ Head and face } \\
\hline Cranial bone & - & $1(0.4)$ \\
\hline Nasal bone & $10(2.1)$ & $7(3.1)$ \\
\hline \multicolumn{3}{|l|}{ Upper limb } \\
\hline Shoulder & $9(1.9)$ & $2(0.9)$ \\
\hline Humerus & $1(0.2)$ & $1(0.4)$ \\
\hline Elbow & $15(3.1)$ & $4(1.7)$ \\
\hline Forearm & $23(4.8)$ & $6(2.6)$ \\
\hline Wrist & $83(17.3)$ & $21(9.1)$ \\
\hline Finger & $194(40.5)$ & $112(48.7)$ \\
\hline \multicolumn{3}{|l|}{ Body trunk } \\
\hline Thoracic cage & $17(3.5)$ & $6(2.6)$ \\
\hline Spine & $7(1.5)$ & $3(1.3)$ \\
\hline Pelvis & $10(2.1)$ & $1(0.4)$ \\
\hline \multicolumn{3}{|l|}{ Lower limb } \\
\hline Femur & $2(0.4)$ & $2(0.9)$ \\
\hline Knee & $10(2.1)$ & - \\
\hline Tibioperoneal bone & $19(4.0)$ & $15(6.5)$ \\
\hline Ankle & $25(5.2)$ & $20(8.7)$ \\
\hline Heel & $1(0.2)$ & $2(0.9)$ \\
\hline Foot & 53 (11.1) & 27 (11.8) \\
\hline
\end{tabular}

Values are expressed as $N(\%)$ order: current exercise habits $(\beta=0.242), \operatorname{BMI}(\beta=0.135)$, and age $(\beta=0.085)$.

\section{Discussion}

Some reports have suggested that the acquisition of bone mass in boys and girls in junior high school is differently influenced by secondary sexual development [7, 9, 14]. It is established that the value of OSI is statistically significantly different between girls who had reached menarche and girls who had not $[2,8,9]$. However, previous studies of the various factors influencing the acquisition of bone mass in junior high school girls did not distinguish between girls who had reached menarche and those who had not. In this study, we investigated the factors influencing bone mass in three groups: boys, girls who had reached menarche, and girls who had not reached menarche.

Comparing junior high school boys, girls who had reached menarche, and girls who had not reached menarche, using ANOVA we noted that there were significant differences in OSI between the three groups. OSI values for girls who had reached menarche were significantly higher than the OSI values for girls who had not reached menarche and for boys aged 12-14 years. Matsueda et al. [15] reported that, in boys aged 10-13 years, the rate of increase in bone strength is highest at the point secondary sex

Table 3 Most common bone fractures and their causes

\begin{tabular}{|c|c|c|c|c|c|c|c|}
\hline & \multirow{2}{*}{$\begin{array}{l}\text { Participants: } \\
\text { Location of bone } \\
\text { fractures* }(N=709) \text { : }\end{array}$} & \multicolumn{3}{|l|}{ Boys } & \multicolumn{3}{|l|}{ Girls } \\
\hline & & $\begin{array}{l}\text { Finger } \\
(N=194)\end{array}$ & $\begin{array}{l}\text { Wrist } \\
(N=83)\end{array}$ & $\begin{array}{l}\text { Foot } \\
(N=53)\end{array}$ & $\begin{array}{l}\text { Finger } \\
(N=112)\end{array}$ & $\begin{array}{l}\text { Foot } \\
(N=27)\end{array}$ & $\begin{array}{l}\text { Wrist } \\
(N=21)\end{array}$ \\
\hline \multicolumn{8}{|l|}{ Sports } \\
\hline Soccer & $93(13.1)$ & $26(13.4)$ & $16(19.3)$ & $16(30.2)$ & $3(2.7)$ & $1(3.7)$ & $1(4.8)$ \\
\hline Tennis & $21(3.0)$ & $2(1.0)$ & $3(3.6)$ & $2(3.8)$ & $2(1.8)$ & $2(7.4)$ & $2(9.5)$ \\
\hline Baseball & $95(13.4)$ & $44(22.7)$ & $8(9.6)$ & $3(5.7)$ & $14(12.5)$ & - & $1(4.8)$ \\
\hline Dodge ball & $27(3.8)$ & $14(7.2)$ & $3(3.6)$ & $2(3.8)$ & $6(5.4)$ & $1(3.7)$ & - \\
\hline Basketball & $80(11.3)$ & $28(14.4)$ & $5(6.0)$ & $1(1.9)$ & $27(24.1)$ & $6(22.2)$ & $1(4.8)$ \\
\hline Volleyball & $47(6.6)$ & $6(3.1)$ & $2(2.4)$ & $2(3.8)$ & $22(19.6)$ & $3(11.1)$ & - \\
\hline Combat sports (judo, karate) & $24(3.4)$ & $4(2.1)$ & $2(2.4)$ & $5(9.4)$ & $2(1.8)$ & $2(7.4)$ & - \\
\hline Kendo & $9(1.3)$ & $4(2.1)$ & - & $4(7.5)$ & - & - & - \\
\hline Athletics & $38(5.4)$ & $4(2.1)$ & $3(3.6)$ & $5(9.4)$ & $2(1.8)$ & $3(11.1)$ & $1(4.8)$ \\
\hline Table tennis & $7(1.0)$ & $2(1.0)$ & $3(3.6)$ & - & - & - & - \\
\hline Other & $52(7.3)$ & $16(8.2)$ & $6(7.2)$ & - & $12(10.7)$ & $3(11.1)$ & $6(28.6)$ \\
\hline Total & $493(69.5)$ & $150(77.3)$ & $51(61.3)$ & $40(75.5)$ & $90(80.4)$ & $21(77.7)$ & $12(57.3)$ \\
\hline Falling down stairs, off bicycles, etc. & $136(19.2)$ & $20(10.3)$ & $21(25.3)$ & $9(17.0)$ & $11(9.9)$ & $4(14.8)$ & $4(19.1)$ \\
\hline Collision with walls or posts & $2(0.3)$ & $1(0.5)$ & - & $1(1.9)$ & - & - & - \\
\hline Fighting & $5(0.7)$ & $2(1.0)$ & $1(1.2)$ & $1(1.9)$ & - & - & - \\
\hline Fingers crushed in gates or doors & $4(0.6)$ & $1(0.5)$ & - & - & $3(2.7)$ & - & - \\
\hline Children's games & $69(9.7)$ & $20(10.3)$ & $10(12.0)$ & $2(3.8)$ & $8(7.1)$ & $2(7.4)$ & $5(23.8)$ \\
\hline
\end{tabular}

Values are expressed as $N(\%)$

* Location of bone fractures is showed in Table 2 


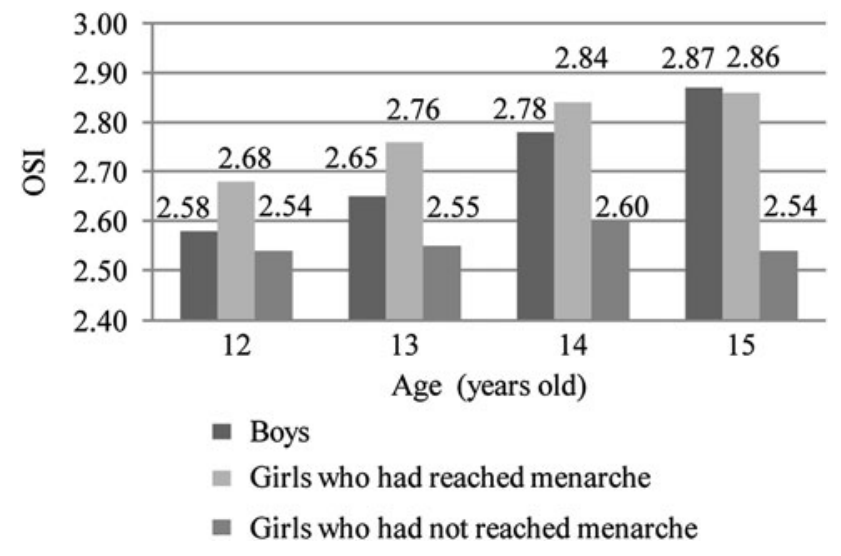

Fig. 1 Average value of OSI according to age and sex

characteristics begin developing; however, for girls aged 9-11 years, the rate of increase is highest just before secondary sex characteristics begin to develop. Because for girls the period of increasing bone mass is earlier than boys, it follows that the OSI values for girls from 12 to 14 years old who had reached menarche were higher than the OSI values for boys of the same age. In addition, during the junior high school years, secondary sexual development begins, resulting in the secretion of an increased level of sex hormones (estrogen, testosterone, etc.). The effect of estrogen on bone mass is to inhibit the formation of osteoclast cells, resulting in reduced bone absorption [16]. The effect of testosterone on bone mass is not only due to increased levels of estrogen (as testosterone is transformed into estrogen) but also due to testosterone directly inhibiting the formation of osteoclast cells, resulting in reduced bone absorption [17]. During junior high school, bone mass is strongly influenced by testosterone in boys, and by estrogen in girls. Accordingly, we would expect the development of secondary sex characteristics to lead to differences in OSI values between boys, girls who had reached menarche, and girls who had not reached menarche.

Table 4 Relationship between five factors and OSI for boys

\begin{tabular}{|c|c|c|c|c|c|c|c|c|}
\hline & \multicolumn{2}{|c|}{12 years old $(N=716)$} & \multicolumn{2}{|c|}{13 years old $(N=1,926)$} & \multicolumn{2}{|c|}{14 years old $(N=1,545)$} & \multicolumn{2}{|c|}{15 years old $(N=787)$} \\
\hline & $N(\%)$ & OSI & $N(\%)$ & OSI & $N(\%)$ & OSI & $N(\%)$ & OSI \\
\hline \multicolumn{9}{|c|}{ Body mass index $\left(\mathrm{kg} / \mathrm{m}^{2}\right)$} \\
\hline-18.4 & $419(58.5)$ & $2.53 \pm 0.24$ & $1,008(52.3)$ & $2.59 \pm 0.23$ & $593(38.4)$ & $2.66 \pm 0.26$ & 209 (26.6) & $2.72 \pm 0.30$ \\
\hline $18.5-24.9$ & $264(36.9)$ & $2.64 \pm 0.25$ & $821(42.6)$ & $2.71 \pm 0.29$ & $866(56.1)$ & $2.85 \pm 0.30$ & $509(64.7)$ & $2.92 \pm 0.35$ \\
\hline $\begin{array}{l}25.0- \\
P \text { value* }\end{array}$ & $33(4.6)$ & $\begin{array}{l}2.79 \pm 0.44 \\
<0.001\end{array}$ & $97(5.0)$ & $\begin{array}{l}2.82 \pm 0.29 \\
<0.001\end{array}$ & $86(5.6)$ & $\begin{array}{l}2.97 \pm 0.35 \\
<0.001\end{array}$ & $69(8.8)$ & $\begin{array}{l}3.03 \pm 0.32 \\
<0.001\end{array}$ \\
\hline \multicolumn{9}{|l|}{ Exercise habits } \\
\hline None & $45(6.3)$ & $2.57 \pm 0.29$ & $118(6.1)$ & $2.57 \pm 0.30$ & $142(9.2)$ & $2.68 \pm 0.31$ & $74(9.4)$ & $2.72 \pm 0.29$ \\
\hline PS or current & $215(30.0)$ & $2.53 \pm 0.23$ & 473 (24.6) & $2.61 \pm 0.23$ & $398(25.8)$ & $2.78 \pm 0.33$ & $212(26.9)$ & $2.86 \pm 0.34$ \\
\hline $\begin{array}{l}\text { PS and current } \\
P \text { value* }\end{array}$ & $456(63.7)$ & $\begin{array}{l}2.60 \pm 0.26 \\
0.006\end{array}$ & $1,335(69.3)$ & $\begin{array}{l}2.67 \pm 0.27 \\
<0.001\end{array}$ & $1,005(65.0)$ & $\begin{array}{l}2.80 \pm 0.29 \\
<0.001\end{array}$ & $501(63.7)$ & $\begin{array}{l}2.90 \pm 0.35 \\
<0.001\end{array}$ \\
\hline \multicolumn{9}{|c|}{ Milk intake per day during PS (mL) } \\
\hline$<200$ & $257(35.9)$ & $2.55 \pm 0.20$ & $705(36.6)$ & $2.63 \pm 0.26$ & $570(36.9)$ & $2.74 \pm 0.31$ & $296(37.6)$ & $2.81 \pm 0.33$ \\
\hline $200-400$ & $289(40.4)$ & $2.59 \pm 0.27$ & $707(36.7)$ & $2.64 \pm 0.26$ & $529(34.2)$ & $2.80 \pm 0.30$ & $280(35.6)$ & $2.88 \pm 0.33$ \\
\hline $\begin{array}{l}>400 \\
P \text { value* }\end{array}$ & $170(23.7)$ & $\begin{array}{l}2.62 \pm 0.29 \\
0.023\end{array}$ & $514(26.7)$ & $\begin{array}{l}2.69 \pm 0.28 \\
<0.001\end{array}$ & $446(28.9)$ & $\begin{array}{l}2.83 \pm 0.31 \\
<0.001\end{array}$ & $211(26.8)$ & $\begin{array}{l}2.94 \pm 0.36 \\
<0.001\end{array}$ \\
\hline \multicolumn{9}{|c|}{ Current milk intake per day (mL) } \\
\hline$<200$ & $272(38.0)$ & $2.57 \pm 0.24$ & $751(39.0)$ & $2.62 \pm 0.26$ & $609(39.4)$ & $2.74 \pm 0.30$ & $299(38.0)$ & $2.83 \pm 0.36$ \\
\hline $200-400$ & $291(40.6)$ & $2.58 \pm 0.25$ & $721(37.4)$ & $2.65 \pm 0.26$ & $547(35.4)$ & $2.80 \pm 0.30$ & $285(36.2)$ & $2.88 \pm 0.32$ \\
\hline $\begin{array}{l}>400 \\
P \text { value* }\end{array}$ & $153(21.4)$ & $\begin{array}{l}2.60 \pm 0.28 \\
0.408\end{array}$ & $454(23.6)$ & $\begin{array}{l}2.69 \pm 0.28 \\
<0.001\end{array}$ & $389(25.2)$ & $\begin{array}{l}2.82 \pm 0.31 \\
<0.001\end{array}$ & $203(25.8)$ & $\begin{array}{l}2.91 \pm 0.33 \\
0.024\end{array}$ \\
\hline \multicolumn{9}{|c|}{ Bone fracture during the preceding year } \\
\hline Yes & $81(11.3)$ & $2.56 \pm 0.21$ & $167(8.7)$ & $2.64 \pm 0.28$ & $131(8.5)$ & $2.77 \pm 0.31$ & $58(7.4)$ & $2.92 \pm 0.33$ \\
\hline $\begin{array}{l}\text { No } \\
P \text { value** }\end{array}$ & 635 (88.7) & $\begin{array}{l}2.58 \pm 0.26 \\
0.351\end{array}$ & $1,759(91.3)$ & $\begin{array}{l}2.65 \pm 0.26 \\
0.571\end{array}$ & $1,414(91.5)$ & $\begin{array}{l}2.78 \pm 0.31 \\
0.557\end{array}$ & 729 (92.6) & $\begin{array}{l}2.87 \pm 0.34 \\
0.250\end{array}$ \\
\hline
\end{tabular}

OSI values are presented as mean \pm standard deviation

OSI osteo-sono assessment index, PS primary school

* One-way analysis of variance, ** Student's $t$ test 


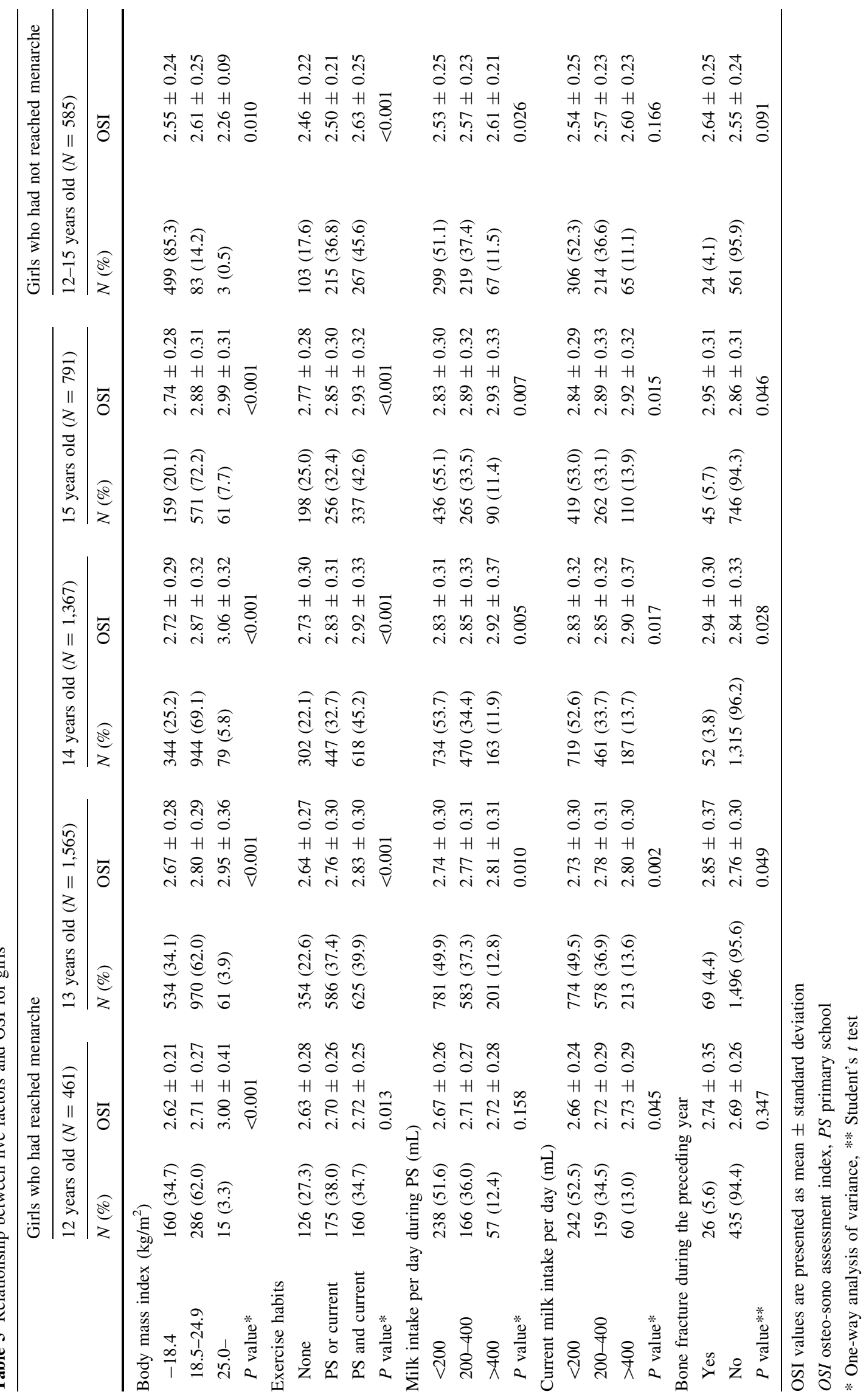


Table 6 Multiple linear regression analysis regarding five factors for OSI

\begin{tabular}{|c|c|c|c|c|c|c|}
\hline & \multicolumn{2}{|c|}{$\begin{array}{l}\text { Boys } \\
(N=4,974)\end{array}$} & \multicolumn{2}{|c|}{$\begin{array}{l}\text { Girls who have reached menarche } \\
(N=4,184)\end{array}$} & \multicolumn{2}{|c|}{$\begin{array}{l}\text { Girls who have not reached menarche } \\
(N=585)\end{array}$} \\
\hline & $\beta$ & $P$ value & $\beta$ & $P$ value & $\beta$ & $P$ value \\
\hline Age & 0.260 & $<0.001$ & 0.140 & $<0.001$ & 0.085 & 0.034 \\
\hline Body mass index & 0.300 & $<0.001$ & 0.302 & $<0.001$ & 0.135 & 0.001 \\
\hline Current exercise habits & 0.106 & $<0.001$ & 0.237 & $<0.001$ & 0.242 & $<0.001$ \\
\hline Milk intake per day in PS & 0.085 & $<0.001$ & 0.039 & 0.083 & 0.114 & 0.141 \\
\hline Current milk intake per day & 0.000 & 0.984 & 0.029 & 0.195 & -0.064 & 0.411 \\
\hline Bone fracture during the preceding year & -0.017 & 0.190 & 0.036 & 0.012 & 0.045 & 0.266 \\
\hline
\end{tabular}

OSI osteo-sono assessment index, $P S$ primary school, $\beta$ standardized partial regression coefficient

Further, our results showed that there were differences between the three groups in the factors that influenced the development of bone mass. MLR showed that, for boys, BMI and age strongly influenced OSI. Current exercise habits and average daily milk intake during primary school influenced OSI to some extent. For girls who had reached menarche, BMI and current exercise habits strongly influenced OSI. Age and bone fracture during the preceding year influenced OSI to some extent. For girls who had not reached menarche, current exercise habits strongly influenced OSI. BMI and age influenced OSI to some extent.

In this study, age had a positive influence on OSI for all three groups. Okano et al. [9] reported that the value of OSI is related to height, weight, and BMI and that, in junior high school students, just as height and weight increase with age, so do BMI and the value of OSI. It is considered that the junior high school years are a very important period for improving physical development such as bone growth.

BMI had a positive influence on OSI for all three groups. Previous studies have indicated a relationship between bone mass and BMI $[2,18]$. Previous studies have also suggested that the continuous stress of body weight on bones increases bone mass [19,20]. Accordingly, because $\mathrm{BMI}$ is one of the factors influencing OSI, maintenance of an adequate BMI value is considered to be important in the acquisition of bone mass.

The influence of daily milk intake in primary school on OSI value was significant only in boys. On the other hand, current milk intake did not influence the OSI value for boys, girls who had reached menarche or girls who had not reached menarche. However, it is generally considered that milk intake is effective in acquiring bone mass. Milk is a calcium-rich beverage and contains approximately $220 \mathrm{mg}$ calcium per $200 \mathrm{~mL}$ [21]. This calcium is in an easily digestible form. Sandler et al. [22] reported that acquiring bone mass was strongly related to calcium intake during growth periods. The Japan Dietetic Association recommends a daily milk intake of 200-400 mL [13]. In particular, the percentage of $0 \mathrm{~mL}$ milk intake per day in the students on days when school lunch was not provided (primary school $36.1 \%$, currently $35.7 \%$ ) was higher than that on days when school lunch was provided (primary school $2.1 \%$, currently $1.1 \%$ ). It is our view that students who drink $<200 \mathrm{~mL}$ of milk per day and only drink milk on days when school lunch is provided might need to increase their consumption of milk.

Current exercise habits had a positive influence on OSI for all three groups; exercise habits increased OSI. Particularly for girls, exercise habits had a great influence on OSI. Ohta et al. [23] suggested that exercise stimuli directly activate bone osteoblast proliferation, resulting in the promotion of osteogenesis, and exercise habits might increase OSI. Indirectly, osteogenesis is promoted by increases in muscle volume and calcium intake and enhanced synthesis of vitamin D in skin as a result of exercising outdoors. The percentage of girls who did not exercise was higher that that of boys. This suggests that establishing good exercise habits is an issue for girls because exercise is one of the factors influencing OSI.

Conversely, for junior high school students, during growth spurts, bone density and bone strength do not increase at the same rate as that of bone growth, resulting in what is known as a period of comparative bone fragility in the long bones of the extremities; exercise during this period of growth has a high risk of resulting in bone fractures [5, 15]. Landin [24] reported that the main cause of bone fractures in junior high school students is not the fragility of the bone material or a problem of bone density, but rather the exercise itself. Our results showed that $69.5 \%$ of bone fractures resulted from sporting activities. Injuries occurring during soccer, baseball, and basketball were the most frequent causes of fractures. There is a concern that bone fractures during the period when bones are growing may pose a problem for peak bone mass (PBM) acquired during the teenage years [6]. In our study, experiencing a bone fracture during the preceding year had a positive effect on OSI for girls who had reached menarche. For girls who had reached menarche, there was a 
significant correlation between bone fracture and exercise habits. Therefore, we consider that the OSI value for girls who had reached menarche was higher not because they had experienced a bone fracture, but because they exercised regularly.

We need to address some of the limitations of this study. First, because our study was a cross-sectional analysis, we were unable to establish causality between influencing factors and the growth of bone mass. Second, the exercise habits questionnaire inquired whether or not junior high school students exercised regularly or played sports, but we were not able to calculate exercise volume or the exertion during exercise. We intend to conduct further longitudinal studies to investigate how bone mass is acquired; these studies will also examine the impact level that exertion and exercise volume have on bone mass acquisition. Further longitudinal studies should be conducted to clarify differences between the factors related to OSI among boys, girls who have reached menarche, and girls who have not reached menarche.

In conclusion, our study showed that there was a difference between the factors that increased OSI among boys, girls who had reached menarche, and girls who had not reached menarche. BMI, exercise habits, and age were the common factors that increased OSI in all three groups. In particular, for girls, exercise had a strong influence on OSI.

We provided the following recommendations to school health programs for the purpose of developing bone mass: (1) all students need to maintain an adequate BMI value, and (2) it is important to promote good exercise habits. In addition, soccer, baseball, and basketball coaches should be aware that these sports have a high incidence of bone fractures and should conduct training accordingly.

Acknowledgments The authors are greatly indebted and grateful to the Ehime Prefecture Board of Education. This study was supported by a grant-in-aid for milk nutrition sponsored by the National Dairy Promotion and Research Association of Japan. The National Dairy Promotion and Research Association plays a major role in developing milk products. In our study, the cost of transporting an acoustic osteoscreener ultrasound device from school to school was covered by a grant-in-aid for milk nutrition from the National Dairy Promotion and Research Association.

Conflict of interest The authors declare that there are no conflicts of interest.

\section{References}

1. Naka H, Iki M, Morita A, Tamaki J, Ikeda Y. A longitudinal study on bone mineral density at lumbar spine and total hip and its determinants in Japanese adolescents-Focus on sports activity at junior high school and high school. Descente Sports Sci. 2005;26:85-94. (article in Japanese).
2. Ito C, Koizumi K, Atsumi K, Suzuki C, Kaneko K. The relationship between ultrasound calcaneal bone mass and lifestyle, physical fitness and body composition in junior high school students. J Jpn Soc Nutr Food Sci. 2007;60(1):53-9. (article in Japanese).

3. Uenishi K, Nakamura K. Intake of dairy products and bone ultrasound measurement in late adolescents: a nationwide crosssectional study in Japan. Asia Pac J Clin Nutr. 2010;19(3):432-9.

4. Noi S, Ozawa H, Koiso T, Masaki T. A study on bone stiffness and related factors in healthy secondary school girls. Jpn J Phys Fit Sports Med. 2000;49:513-22. (article in Japanese).

5. Hagino $\mathrm{H}$, Yamamoto $\mathrm{K}$, Ohshiro $\mathrm{H}$, Nose T. Increasing incidence of distal radius fractures in Japanese children and adolescents. J Orthop Sci. 2000;5(4):356-60.

6. Seino Y. How to increase bone mass during childhood. Nihonrinsho. 2007;65(925):623-7. (article in Japanese).

7. Kokudo S, Matsumoto K. Bone condition as detected by sound transmission test of bones in adolescence and effects of exercise on the condition. Adolescentology. 1997;15(3):279-85. (article in Japanese).

8. Hayashi K, Nakajima K, Takami S, Hashitani T. The relationship between bone mineral density and lifestyle of junior high school. J Jpn Red Cross Toyota Coll Nurs. 2011;6(1):47-53. (Article in Japanese).

9. Okano R. The growth characteristic and sexual difference of calcaneal bone stiffness. Jpn J Sch Health. 2004;46(1):59-66. (article in Japanese).

10. Gaccom. Directory of elementary and junior high schools in Japan. http://www.gaccom.jp/search/p38/school_meal/. Accessed 10 Aug 2012.

11. Heaney RP, Avioli LV, Chesnut CH, Lappe J, Recker RR, Brandenburger GH. Ultrasound velocity, through bone predicts incident vertebral deformity. J Bone Miner Res. 1995;10(3): $341-5$.

12. Matsuzawa Y, Inoue S, Ikeda Y, Sakata T, Saito Y, Sato U, et al. Atarashii himan no hantei to himanshou no shindan kijun (New diagnostic criteria for obesity and obesity related disorder). J Jpn Soc Study Obes. 2000;6:18-28. (article in Japanese).

13. The Japan Dietetic Association. Nutritional guidance questions and answers. http://www.dietitian.or.jp/consultation/e_02.html. Accessed 10 Aug 2012.

14. Naka H, Iki M, Morita A, Ikeda Y. Effects of pubertal development, height, weight, and grip strength on the bone mineral density of the lumbar spine and hip in peripubertal Japanese children: Kyoto kids increase density in the skeleton study (Kyoto KIDS study). J Bone Miner Metab. 2005;23(6):463-9.

15. Matsueda M, Takahashi K, Kaga M, Moriwake T, Seino Y. Effects of growth rate and life-style on bone strength. Jpn J Sch Health. 2001;42(6):486-95. (article in Japanese).

16. Hughes DE, Dai A, Tiffee JC, Li HH, Mundy GR, Boyce BF. Estrogen promotes apoptosis of murine osteoclasts mediated by TGF-beta. Nat Med. 1996;2(10):1132-6.

17. Kawano H, Sato T, Yamada T, Matsumoto T, Sekine K, Watanabe T, et al. Suppressive function of androgen receptor in bone resorption. PNAS. 2003;100:9416-21.

18. Nagamasu $T$, Tugawa $K$, Asai $H$. Investigation study about a bone evaluation instrumentation value in junior high school students. Osaka Kyoiku Univ Repos. 2008;56(2):27-39. (article in Japanese).

19. Edelstein SL, Barrett-Connor E. Relation between body size and bone mineral density in elderly men and women. Am J Epidemiol. 1993;138(3):160-9.

20. Frost HM. Suggested fundamental concepts in skeletal physiology. Calcif Tissue Int. 1993;52(1):1-4.

21. The Ministry of Education, Culture, Sports, Science and Technology. Food Composition Database. http://fooddb.jp/index.pl. Accessed 10 Aug 2012. 
22. Sandler RB, Slemenda CW, LaPorte RE, Cauley JA, Schramm MM, Barresi ML, et al. Postmenopausal bone density and milk consumption in childhood and adolescence. Am J Clin Nutr. 1985;42(2):270-4.

23. Ohta H, Fuyuki T, Sugimoto I, Makita K, Takamatsu K, Nozawa S. Kotsusoshousho no yobohou (The method of preventing osteoporosis). Obstet Gynecol Ther. 1996;72:1083-8. (article in Japanese).

24. Landin LA. Fracture patterns in children. Analysis of 8,682 fractures with special reference to incidence, etiology and secular changes in a Swedish urban population 1950-1979. Acta Orthop Scand Suppl. 1983;202:1-109. 